\title{
Full QCD calculation of neutron electric dipole moment with the external electric field method
}

\author{
E. Shintani, ${ }^{1}$ S. Aoki, ${ }^{2,3}$ and Y. Kuramashi ${ }^{2,4}$ \\ ${ }^{1}$ High Energy Accelerator Research Organization (KEK), Tsukuba 305-0801, Japan \\ ${ }^{2}$ Graduate School of Pure and Applied Sciences, University of Tsukuba, Tsukuba, Ibaraki 305-8571, Japan \\ ${ }^{3}$ Riken BNL Research Center, Brookhaven National Laboratory, Upton, New York 11973, USA \\ ${ }^{4}$ Center for Computational Sciences, University of Tsukuba, Tsukuba, Ibaraki 305-8577, Japan
}

(Received 6 March 2008; published 16 July 2008)

\begin{abstract}
We have calculated the neutron electric dipole moment (EDM) in the presence of the $C P$ violating $\theta$ term in lattice QCD with two-flavor dynamical clover quarks, using the external electric field method. Accumulating a large number of statistics by the averages over 16 different source points and over forward and backward nucleon propagators, we have obtained nonzero signals of neutron and proton EDM beyond 1 standard deviation at each quark mass in full QCD. We have investigated the quark mass dependence of nucleon EDM in full QCD, and have found that nucleon EDM in full QCD does not decrease toward the chiral limit, as opposed to the theoretical expectation. We briefly discuss possible reasons for this behavior.
\end{abstract}

DOI: 10.1103/PhysRevD.78.014503

PACS numbers: 12.38.Gc, 11.30.Er, 11.30.Rd, 12.39.Fe

\section{INTRODUCTION}

A requirement for renormalizability in QCD allows a $C P$ violating term with a free parameter $\theta_{\mathrm{QCD}}$, thus called the $\theta$ term,

$$
\mathcal{L}_{\delta C P}=\theta_{\mathrm{QCD}} \frac{g^{2}}{32 \pi^{2}} G_{\mu \nu} \tilde{G}^{\mu \nu},
$$

where $G_{\mu \nu}$ and $\tilde{G}_{\mu \nu}$ represent a gluon field strength and its dual, and $g$ is a coupling constant of QCD. If the $C P$ invariance is preserved in the strong interaction in nature, $\theta_{\mathrm{QCD}}$ must be zero in QCD. Indeed a current experimental bound of the neutron electric dipole moment (NEDM) [1],

$$
d_{N}<6.3 \times 10^{-13} \mathrm{e} \cdot \mathrm{fm},
$$

and the crude theoretical estimates [2-4], $d_{N}=$ $\mathcal{O}\left(10^{-2}\right) \theta_{\mathrm{QCD}}$, gives the constraint that $\theta_{\mathrm{QCD}} \lesssim$ $\mathcal{O}\left(10^{-10}\right) . \theta_{\mathrm{QCD}}$ must be very small or even zero.

In the presence of the electroweak interaction, however, the above conclusion on $\theta_{\mathrm{QCD}}$ is modified. In the Weinberg-Salam model of the electroweak interaction, the quark mass term generated through Yukawa couplings by the spontaneous electroweak symmetry breaking is given by

$$
\mathcal{L}_{\mathrm{m}}=\bar{q}_{L}^{i}\left(M_{\mathrm{CKM}}\right)_{i j} q_{R}^{j}+\text { H.c., }
$$

with a quark mass matrix $M_{\mathrm{CKM}}$ and left- and right-handed quark fields $q_{R, L}$. In order to transform the quark mass matrix to a real and diagonal form, $\mathrm{U}(1)$ as well as $\mathrm{SU}\left(N_{f}\right)$ chiral rotations are necessary, since $M_{\mathrm{CKM}}$ is nonHermitian in general. Through the Adler-Bell-Jackiw anomaly, this $\mathrm{U}(1)$ chiral transformation shifts $\theta_{\mathrm{QCD}}$ in Eq. (1) to

$$
\bar{\theta}=\theta_{\mathrm{QCD}}+\arg \operatorname{det} M_{\mathrm{CKM}} .
$$

Therefore the experimental bound (2) on $d_{N}$ leads to $\bar{\theta} \lesssim$ $\mathcal{O}\left(10^{-10}\right)$ : A subtle cancellation between a parameter in $\mathrm{QCD}\left(\theta_{\mathrm{QCD}}\right)$ and a phase of mass matrix in the WeinbergSalam model ( $\arg \operatorname{det} M_{\mathrm{CKM}}$ ) should be fulfilled to keep the $C P$ invariance in the strong interaction. This cancellation seems unnatural and thus a new mechanism must exist to explain the smallness of $\bar{\theta}$ ("strong $C P$ problem").

The simplest solution to the strong $C P$ problem is that one of the quarks is massless, so that we can set $\bar{\theta}=0$ by the chiral rotation of this quark without introducing complex phases to other quark masses. Detailed analyses by chiral perturbation theory [5] or lattice QCD [6], however, strongly indicate that the up quark has a finite mass $(1.5<$ $m_{u}<3.0 \mathrm{MeV}$ [7]), therefore this solution is excluded. In the Peccei-Quinn (PQ) mechanism [8], $\bar{\theta}$ is promoted to a scalar field associated with a new symmetry, called PQ symmetry, which is slightly broken by the anomaly. The spontaneous PQ symmetry breaking automatically picks up a unique vacuum, in which $\bar{\theta}$ vanishes. As a consequence of the symmetry breaking, a very light new scalar particle, called axion must exist in this mechanism. So far the axion, which is also one of the candidates for the cold dark matter, has not been observed yet, and both cosmological observations and accelerator experiments set a very narrow allowed region of the axion mass such that $10^{-6}<$ $m_{a}<3 \times 10^{-3} \mathrm{eV}$ [9].

An aim of our investigation in this paper is not to propose a new solution to the strong $C P$ problem, but is to establish a reliable way of calculating the NEDM in lattice QCD. For small $\bar{\theta}$, the NEDM is proportional to $\bar{\theta}$ as $d_{N}=d_{N}^{(1)} \bar{\theta}+O\left(\bar{\theta}^{3}\right)$. Various model calculations [2,3] lead to different estimates, ranging that $\left|d_{N}^{(1)}\right|=\mathcal{O}\left(10^{-2} \sim\right.$ $\left.10^{-3}\right) \mathrm{e} \cdot \mathrm{fm}$, and even its sign has not been determined yet. The lattice QCD has a potential to calculate $d_{N}^{(1)}$ nonperturbatively and in a model independent way. Once a 
method of calculating the NEDM is established in lattice QCD for the case of a particular $C P$ violating term given in (1), it may become possible to extend NEDM calculations to the case of new $C P$ violating terms such as the chromoelectric dipole moment [4] generated in supersymmetry models at high energy [10].

Lattice calculations of $d_{N}^{(1)}$ have remained to be notoriously difficult for a long time [11]. In our previous papers $[12,13]$ we have investigated two methods of calculating $d_{N}^{(1)}$ in quenched lattice QCD. In the first method [12], we have calculated the NEDM form factor at nonzero momentum transfer, which becomes the NEDM in the limit of zero momentum transfer. In the second method, the NEDM has been directly extracted from the energy shift in the presence of the external electric field. In both cases, we have successfully obtained nonzero signals of EDM for neutrons and protons. In this paper, we extend the calculation of the NEDM to the case of two-flavor full QCD, using configurations generated by the CP-PACS collaboration [14]. We employ the second method, the direct calculation of the NEDM with the external electric field, since no extrapolation of momentum transfer is needed, as opposed to the form factor method. This method, however, requires a large number of configurations to reduce statistical errors. Performing calculations at four different quark masses, we discuss a quark mass dependence of the NEDM in full QCD. In particular it is interesting to see whether the NEDM vanishes or not at zero quark mass as theoretically predicted. There exists a previous study of the NEDM in lattice QCD with two-flavor dynamical domain-wall quarks using the form factor method [15]. Unfortunately, the signal of the NEDM is consistent with zero within a large error, so that only an upper bound is obtained for a value of $d_{N}^{(1)}$.

The organization of this paper is as follows. In Sec. II, we give a definition of NEDM in the presence of uniform and static electric fields. We then consider how this external electric field is introduced on the lattice, and discuss a violation of uniformity of the electric field due to boundaries of the finite lattice. We also explain our method of extracting the NEDM from the nucleon propagator. In Sec. III, simulation parameters for dynamical configurations are briefly summarized. Our main results are given in Sec. IV. Summary and discussions of this paper are presented in Sec. V. In this paper, we set $a=1$ unless necessary.

\section{METHOD OF THE NEDM CALCULATION}

\section{A. Definition of the NEDM}

In the presence of the constant and uniform electric field $\vec{E}$, a change of energy for the nucleon state due to the $\theta$ term is denoted as

$$
\Delta \mathcal{E}_{C P}=d_{N}^{(1)} \theta \vec{S} \cdot \vec{E}+\mathcal{O}\left(\vec{E}^{3} \theta, \vec{E} \theta^{3}\right)
$$

with the nucleon spin vector $\vec{S}$. This leads to the following extraction of $d_{N}^{(1)}$ for $\vec{E}=\left(0,0, E_{z}\right)$ :

$$
\mathcal{E}_{+}^{\theta}\left(E_{z}\right)-\mathcal{E}_{-}^{\theta}\left(E_{z}\right)=d_{N}^{(1)} \theta E_{z}+\mathcal{O}\left(E_{z}^{3} \theta, E_{z} \theta^{3}\right)
$$

where $\mathcal{E}_{ \pm}^{\theta}\left(E_{z}\right)$ is an energy of the nucleon state with $S_{z}=$ $\pm 1 / 2$ in the presence of the electric field $\vec{E}=\left(0,0, E_{z}\right)$. Hereafter we simply denote $d_{N}^{(1)}$ as $d_{N}$.

\section{B. Introduction of the electric field on the lattice and a boundary effect}

On the lattice, an external electric field $E_{k}$ is introduced into linked variables via a replacement in the Wilson-Dirac operator with

$$
\begin{aligned}
U_{k}(x) \rightarrow e^{e_{q} E_{k} t} U_{k}(x) & \equiv U_{k}^{q}(E, x), \\
U_{k}^{\dagger}(x) \rightarrow e^{-e_{q} E_{k} t} U_{k}^{\dagger}(x) & \equiv \bar{U}_{k}^{q}(E, x),
\end{aligned}
$$

where $e_{q}$ is an electric charge of a quark flavor $q$. Note here that a complex factor $i$ does not appears in the exponent since $E_{k}$ is defined in the "Minkowski" space while $t$ is the time coordinate of the "Euclidean" lattice, and therefore $U_{k}^{q}(E, x)$ is no more unitary. ${ }^{1}$ In our calculation we have made this replacement of the link variables only for the valence quark [13], while gauge configurations have been already generated without this replacement in the quark determinant. This "approximation" is equivalent to ignoring the "disconnected" contribution from the electric field. It is noted that, at the first order of the electric field, this contribution vanishes for the three-flavor QCD with $m_{u}=$ $m_{d}=m_{s}$, since the disconnected contribution does note depend on the quark flavor in the flavor SU(3) limit, and therefore is proportional to $e_{u}+e_{d}+e_{s}=0$ after summing over three flavors.

As discussed in Ref. [13], the introduction of the Minkowski electric field destroys the periodic boundary condition of link variable $U_{k}^{q}(E, x)$, so that translational invariance of the electric field is violated at the temporal boundary. Indeed an effective electric field, defined by $E_{k}\left(t_{E}\right)=\left\{\ln U_{k}^{q}\left(E, t_{E}+1\right)-\ln U_{k}^{q}\left(E, t_{E}\right)\right\} / e_{q}$ with $U_{k}\left(t_{E}\right)=$ 1 , becomes

$$
E_{k}\left(t_{E}\right)=\left\{\begin{array}{ll}
E_{k} & \text { at } t_{E}=1,2, \cdots T-1, \\
-(T-1) E_{k} & \text { at } t_{E}=T
\end{array},\right.
$$

where $t_{E}$ runs from one to $T$, so that $t_{E}=T+1$ is equal to $t_{E}=1$. A strong antielectric field is generated between $t_{E}=1$ and $t_{E}=T$ in order to cancel the constant electric field $E_{k}$, so that $\sum_{t_{E}=1}^{T} E_{k}\left(t_{E}\right)$ vanishes. We denote the place where the large gap exists as $t_{\mathrm{Gap}}$, and $t_{\mathrm{Gap}}=0$ in the above case. Since the definition of the NEDM in (6) requires the

\footnotetext{
${ }^{1}$ With the electric field in the Minkowski space, however, the energy difference (6) becomes real in the lattice calculation, so that it can be extracted from the ratio nucleon propagators between spin-up and spin-down [11].
} 
FULL QCD CALCULATION OF NEUTRON ELECTRIC ...

constant electric field, the NEDM should only be measured far away from the boundary to avoid an effect of the antielectric field. In this work we keep distances between source/sink points and the boundary as large as possible. In particular we fix $\left|t_{\mathrm{Gap}}-t_{\mathrm{src}}+1\right|=T / 2$, where $t_{\mathrm{src}}$ is the time coordinate of the source point.

In the previous study [13] we found that the good sampling of the topological charge is important for obtaining the reliable signal of NEDM. At least an order of a few thousands configurations was needed to realize the symmetric and Gaussian distribution in quenched QCD. On the other hand, a number of full QCD configurations in this study is limited to $700 \sim 750$ at each quark mass. We therefore use several source points for each configuration to increase statistics. In accordance with the change of the source point, we shift the boundary point, keeping the distance between the source point and the boundary as large as possible, to avoid the boundary effect mentioned above.

\section{Spinor structure of the nucleon propagator and EDM}

In the presence of the electric field $\vec{E}$ and the $C P$ violating $\theta$ term, the explicit form of the nucleon propagator becomes

$$
\begin{aligned}
\left\langle N_{\alpha} \bar{N}_{\beta}\right\rangle_{\theta}(\vec{E}, t)= & Z_{N}\left(E^{2}\right)\left[\left(1+A_{N}\left(E^{2}\right) \theta \vec{\sigma} \cdot \vec{E}\right)\right. \\
& \left.\times \exp \left(-\mathcal{E}_{N}\left(E^{2}\right) t-\frac{d_{N} \theta}{2} \vec{\sigma} \cdot \vec{E} t\right)\right]_{\alpha \beta} \\
& +\cdots
\end{aligned}
$$

with an overall amplitude $Z_{N}\left(E^{2}\right)$, a coefficient $A_{N}\left(E^{2}\right)$, and an energy $\mathcal{E}_{N}\left(E^{2}\right){ }^{2}$ The ellipse represents $\mathcal{O}\left(E^{3} \theta, \theta^{2}\right)$ terms and contributions from excited states. Here $\langle\mathcal{O}\rangle_{\theta}$ represents a vacuum expectation value in the reweighting method as

$$
Z_{\theta}\langle\mathcal{O}\rangle_{\theta}=Z_{\theta=0}\left\langle\mathcal{O} e^{i \theta Q}\right\rangle_{\theta=0}
$$

with topological charge $Q$, which is evaluated by the cooling method in our calculation $[12,13]$.

In order to extract $d_{N}$ from the nucleon propagator in the above equation, we consider the following ratio between different spinor components:

$$
\begin{aligned}
R_{3}(E, t ; \theta) & =\frac{R_{3}^{\text {naive }}(E, t ; \theta)}{R_{3}^{\text {naive }}(E=0, t ; \theta)} \frac{R_{3}^{\text {naive }}(E=0, t ; \theta=0)}{R_{3}^{\text {naive }}(E, t ; \theta=0)} \\
& \simeq \frac{1+\theta A_{N}\left(E^{2}\right) E}{1-\theta A_{N}\left(E^{2}\right) E} \exp \left[-d_{N} \theta E t\right]
\end{aligned}
$$

where

\footnotetext{
${ }^{2}$ Note that because of the acceleration for a charged particle the energy of proton increases as a time increases [16]. However this contribution is canceled out in the following ratio.
}

$$
\begin{aligned}
R_{3}^{\text {naive }}(E, t ; \theta) & =\frac{\left\langle N_{1} \bar{N}_{1}\right\rangle_{\theta}((0,0, E), t)}{\left\langle N_{2} \bar{N}_{2}\right\rangle_{\theta}((0,0, E), t)} \\
& =\frac{1+A_{N} \theta\left(E^{2}\right) E}{1-A_{N} \theta\left(E^{2}\right) E} \exp \left[-d_{N} \theta E t\right]+\cdots .
\end{aligned}
$$

Three additional $R_{3}^{\text {naive's }}$ in $R_{3}(E, t ; \theta)$ are introduced to remove contamination coming from $\theta=0$ and/or $E=0$ terms due to the insufficient statistics. In addition, to remove fictitious $E^{2 n} \theta$ contribution, we construct the ratio

$$
\begin{aligned}
R_{3}^{\text {corr }}(E, t ; \theta) & =\frac{R_{3}(E, t ; \theta)}{R_{3}(-E, t ; \theta)} \\
& =\frac{R_{3}^{\text {naive }}(E, t ; \theta)}{R_{3}^{\text {naive }}(-E, t ; \theta)} \frac{R_{3}^{\text {naive }}(-E, t ; \theta=0)}{R_{3}^{\text {naive }}(E, t ; \theta=0)} \\
& \simeq\left(\frac{1+\theta A_{N}^{1}\left(E^{2}\right) E}{1-\theta A_{N}^{1}\left(E^{2}\right) E}\right)^{2} \exp \left[-2 d_{N} \theta E t\right],
\end{aligned}
$$

with $R_{3}(E, t ; \theta)$ in Eq. (11). For later use we define the "effective $d_{N}$ " as

$$
2 d_{N} \theta E=\ln \left[\frac{R_{3}^{\operatorname{corr}}(E, t-1 ; \theta)}{R_{3}^{\operatorname{corr}}(E, t ; \theta)}\right]
$$

which is validated in large $t$ where the nucleon asymptotic state dominates.

\section{SIMULATION PARAMETERS}

In our calculation, we employ two-flavor dynamical QCD configurations, generated by the CP-PACS collaboration [14] with the renormalization group (RG)-improved (Iwasaki) gauge action and the clover quark action on $24^{3} \times 48$ lattice at $\beta=2.1$. The corresponding lattice spacing, determined by the rho meson mass $m_{\rho}=$ $768.4 \mathrm{MeV}$, is $a^{-1} \simeq 1.8 \mathrm{GeV}(a \simeq 0.11 \mathrm{fm})$.

The valence quark mass is chosen to be same with the sea quark mass. The pion mass $m_{\mathrm{PS}}$ becomes 1.13, 0.93, 0.76 , and $0.53 \mathrm{GeV}$ at $K_{\text {sea }}=0.1357,0.1367,0.1374$, and 0.1382 , respectively. Table I lists lattice parameters used in our simulation. Throughout this paper statistical errors are estimated by the jackknife method, whose bin size is five configurations, equivalent to 25 hybrid Monte Carlo (HMC) trajectories. We employ a local sink and a smeared source for all three quark propagators in the nucleon twopoint function, with the exponential smearing, $f(r)=$ $A e^{-B r}$. Parameters $(A, B)$ depend on the quark mass as shown in Table I.

We take 16 different source points separated by three lattice units in temporal direction and maximally separated in spatial directions. Averaging these source points, a total number of statistics is more than 10000 .

Throughout our study, the value of the electric field and $\theta$ are fixed to $E=0.004$ and $\theta=0.025$, which are the most suitable choice to reduce statistical errors [13]. 
TABLE I. Table for the simulation parameters in this work. $\mathcal{O}(a)$ improved coefficient of the clover term $c_{S W}$ is chosen as $c_{S W}=$ 1.47. The column of $(A, B)$ denotes the smeared source parameters, and $K$ denotes the hopping parameter for the degenerate up and down quarks.

\begin{tabular}{lcccccc}
\hline \hline Lattice size & Physical volume $\left(\mathrm{fm}^{3}\right)$ & Cutoff $a^{-1}(\mathrm{GeV})$ & $K$ & $(A, B)$ & $m_{\mathrm{PS}} / m_{V}$ & $m_{N} a$ \\
\hline $24^{3} \times 48$ & $2.6^{3}$ & 1.83 & 0.1357 & $(1.5,0.45)$ & 0.81 & $1.1851(10)$ \\
& & & 0.1367 & $(1.5,0.43)$ & 0.76 & $1.0224(11)$ \\
& & & 0.1374 & $(1.5,0.35)$ & 0.69 & $0.8944(12)$ \\
& & 0.1382 & $(1.5,0.25)$ & 0.58 & $0.7167(14)$ \\
\hline \hline
\end{tabular}

\section{RESULTS}

\section{A. Topological charge distribution and nucleon mass}

We measure the topological charge of each configuration after 50 cooling steps, using the $\mathcal{O}\left(a^{2}\right)$ improved definition of the topological charge density given by

$$
\begin{aligned}
Q_{\text {improved }}= & \frac{1}{32 \pi^{2}} \varepsilon_{\mu \nu \alpha \beta}\left(a_{0} \operatorname{Tr}\left[L_{\mu \nu}^{1 \times 1} L_{\alpha \beta}^{1 \times 1}\right]\right. \\
& \left.+2 a_{1} \operatorname{Tr}\left[L_{\mu \nu}^{1 \times 2} L_{\alpha \beta}^{1 \times 2}\right]\right)
\end{aligned}
$$

with $a_{0}=5 / 3$ and $a_{1}=-1 / 12[17,18]$, where $L_{\mu \nu}^{1 \times 1}$ and $L_{\mu \nu}^{1 \times 2}$ are $1 \times 1$ and $1 \times 2$ Wilson loops, respectively. We show the time history of the topological charge in Fig. 1 and its histogram in Fig. 2 at four quark masses. The distributions of the topological charge is more or less Gaussian at all quark masses. As the sea quark mass decreases, the width of the Gaussian distribution becomes narrower in accordance with the theoretical expectation.

The effective mass of the nucleon at four quark masses is plotted in Fig. 3. The average over 16 sources gives enough
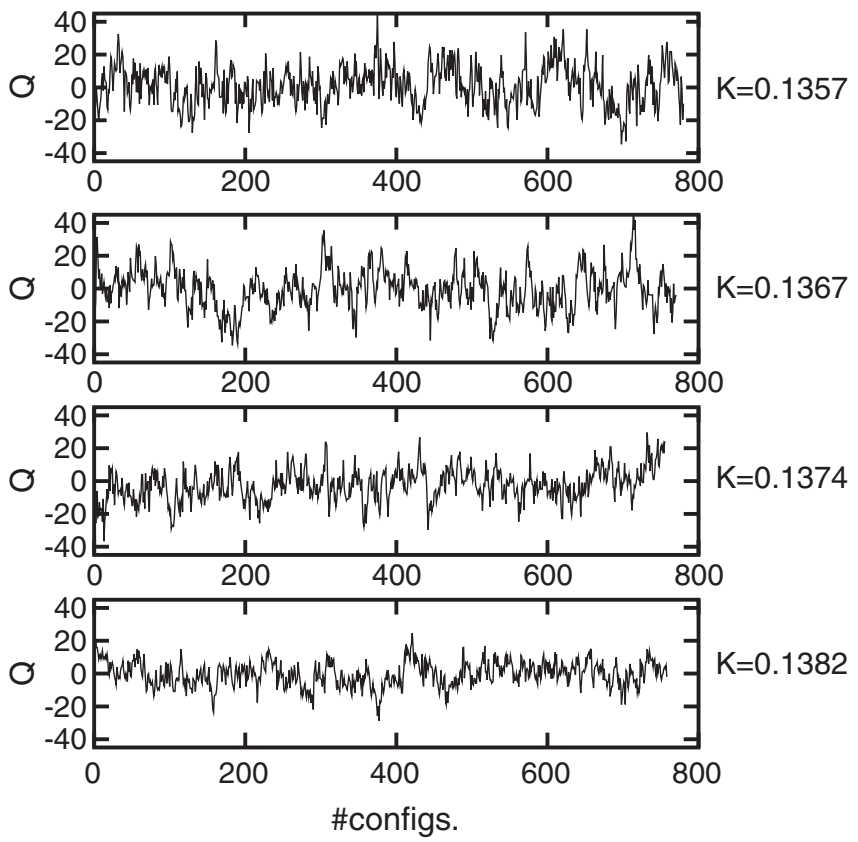

FIG. 1. Time histories of the topological charge at each sea quark mass. statistics to produce a clear plateau at all cases. We see that the plateau for the nucleon state starts at $t-t_{\mathrm{src}}+1=6$. The global fit of the propagator from $t-t_{\mathrm{src}}+1=9$ to 15 gives a value of the nucleon mass with a very small error, as shown in Table I.

\section{B. Signal of EDM}

In the previous study we found that the influence due to the gap of the electric field on the EDM signal disappears at $\left|t_{\text {Gap }}-t_{\text {src }}+1\right| \geq 5$ at $a=0.1 \mathrm{fm}$ in quenched QCD [13]. Since we fix $\left|t_{\text {Gap }}-t_{\text {src }}+1\right|=T / 2=24$ in this study, the above condition is well satisfied. In this setup, the backward propagation of the nucleon becomes identical to the forward propagation in the infinite statistics, so that we can take an average over both to increase statistics. In Fig. 4, we compare the time dependence of $R_{3}(E, t ; \theta)$ between forward and backward propagation for neutrons and protons. We have found that two results marginally agree with each other at $t-t_{\mathrm{src}}+1 \leq 10$. Here we consider that the difference at $t-t_{\mathrm{src}}+1>10$ is caused by the statistical noises, and thus the signal of EDM is obtained only at $t-$ $t_{\mathrm{src}}+1 \leq 10$. Therefore a fitting range should be chosen as
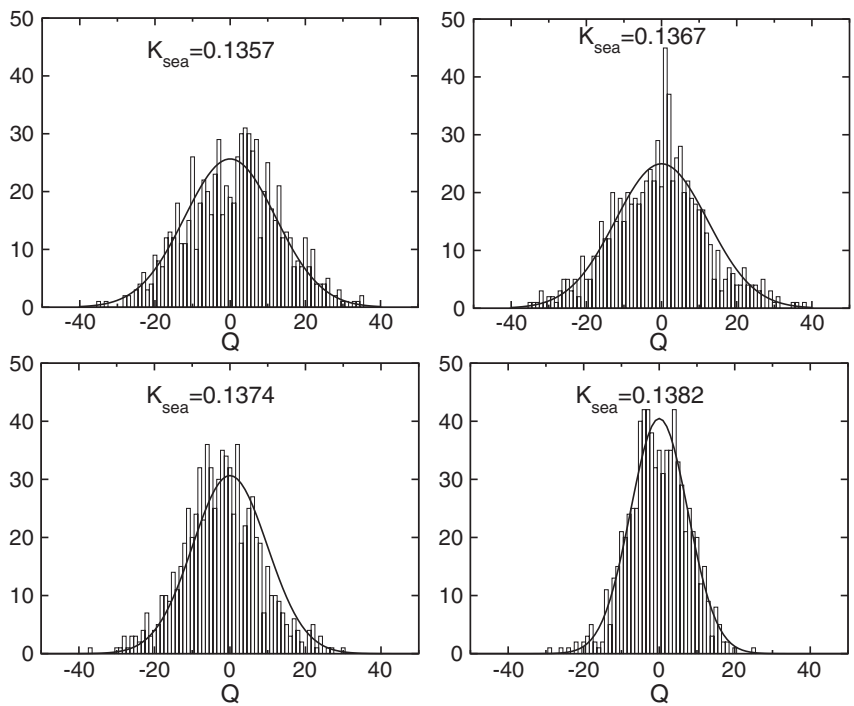

FIG. 2. Histograms of the topological charge at each sea quark mass. The solid line denotes the Gaussian distribution determined from $\langle Q\rangle$ and $\left\langle Q^{2}\right\rangle$. 
FULL QCD CALCULATION OF NEUTRON ELECTRIC ...

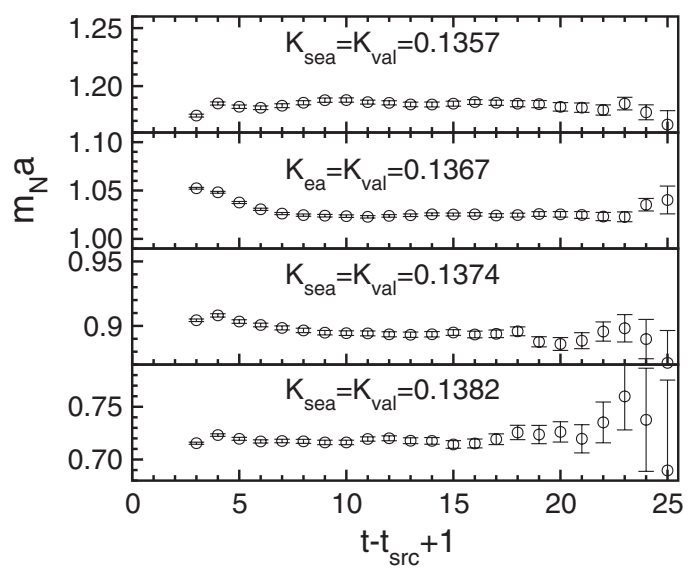

FIG. 3. The nucleon effective mass as a function of time in lattice unit without electric field. The average over 16 source sets on each configuration is taken.

$6 \leq t-t_{\mathrm{src}}+1 \leq 10$. Since $T-\left(t-t_{\mathrm{Gap}}\right)=T / 2-9=$ 15 , the distance between the gap and the end point of the fitting ranged at $t-t_{\mathrm{src}}+1=10$, is much larger than five, the gap of the electric field does not affect the EDM signal.

In Figs. 5-8, the time dependence of $R_{3}(E, t ; \theta)$ with $E= \pm 0.004$ is plotted for the neutron and proton at each quark mass. At all quark masses, the signal for nonzero EDM can be seen at $6 \leq t-t_{\mathrm{src}}+1 \leq 10$, and the signal changes its sign as $E$ does. To estimate the size of nucleon EDM, we consider an effective mass of $R_{3}^{\text {corr }}(E, t ; \theta)$ defined in Eq. (14), and plot it in Figs. 9-12. Although errors and fluctuations are large, nonzero signals for both proton
PHYSICAL REVIEW D 78, 014503 (2008)
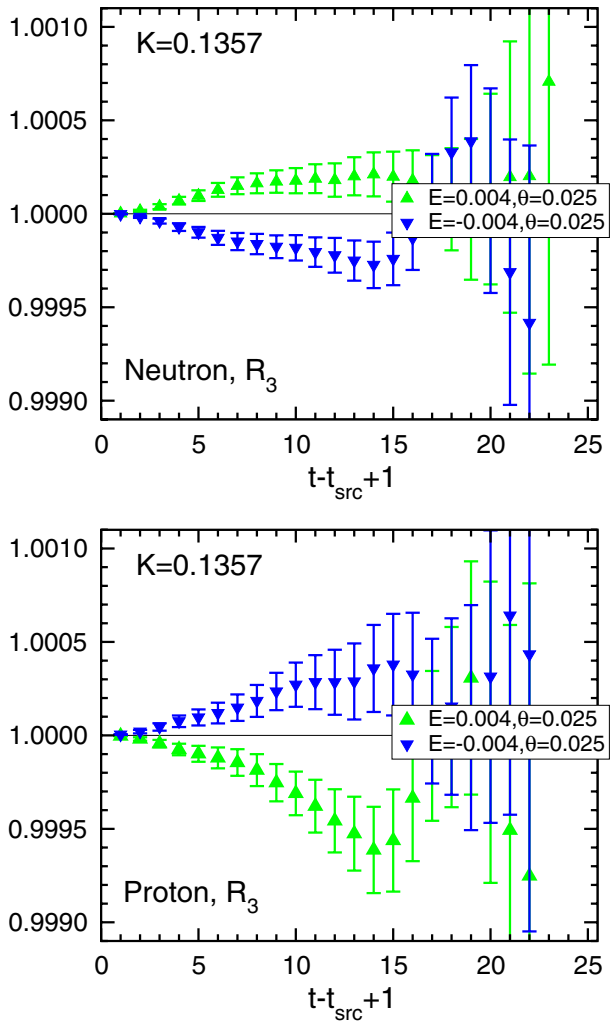

FIG. 5 (color online). $\quad R_{3}$ as a function of time at $K=0.1357$, after averaging 16 source sets $\left(t_{\text {src }}=3,6, \cdots, 48\right)$, with $E=$ \pm 0.004 and $\theta=0.025$ for neutrons (top) and protons (bottom). The different symbols denote the different signs of the electric field.
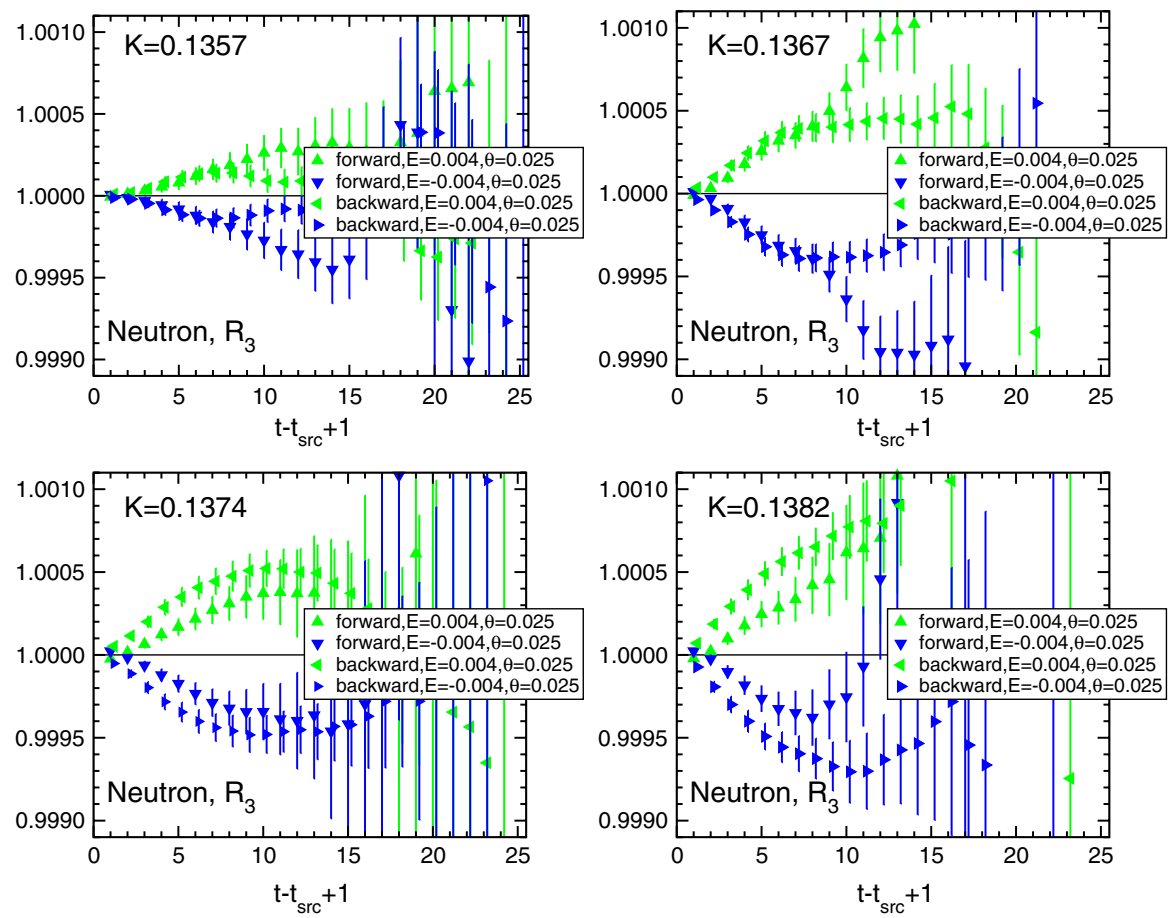

FIG. 4 (color online). Comparison between forward and backward propagation of $R_{3}$ for neutron as a function of time at $E=$ \pm 0.004 and $\theta=0.025$. The average over 16 source sets $\left(t_{\mathrm{src}}=3,6, \cdots, 48\right)$ is taken. 

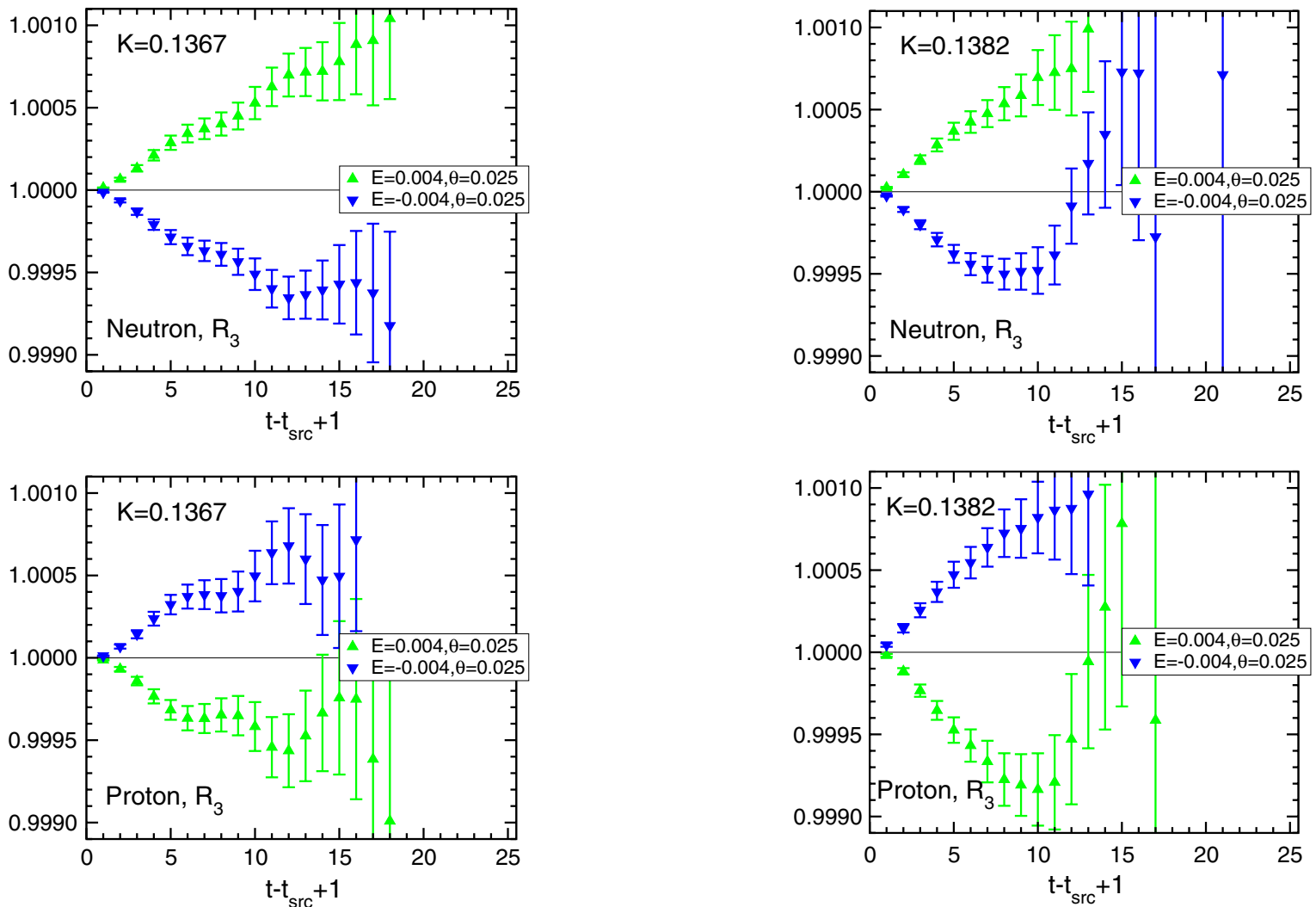

FIG. 6 (color online). Same as Fig. 5 at $K=0.1367$.

FIG. 8 (color online). Same as Fig. 5 at $K=0.1382$.

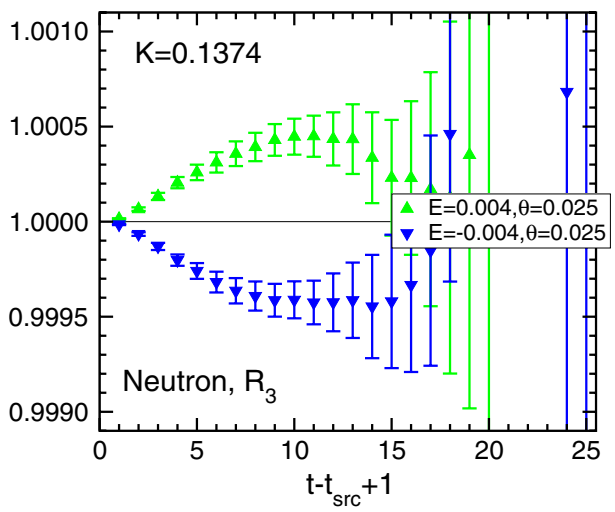

and neutron EDM have been observed in full QCD simulations for the first time. By fitting $R_{3}^{\text {corr }}(E, t ; \theta)$ with an exponential function in Eq. (13) at $6 \leq t-t_{\text {src }}+1 \leq 10$, we obtain the value of $d_{N}^{(1)}$, which is given in Table II. Signs of EDM are opposite between protons and neutrons. This agrees with the quenched result [13] and with the chiral perturbation theory prediction [3].

\section{Mass dependence of the nucleon EDM}

In Fig. 13, $d_{N}$ for neutrons and protons are plotted as a function of pion mass squared, $m_{\mathrm{PS}}^{2}$, together with the quenched results. Unfortunately, because of large statistical errors in full QCD results, it is hard to observe a difference from the quenched results. Compared with the model calculation [2], the central value is 10 times larger although its errors are large.

Mass dependence of $d_{N}$ in full QCD does not show the expected decrease toward the chiral limit. There are several possible explanations. First, large statistical errors might hide the actual decrease of $d_{N}$ toward the chiral limit. Second, the quark mass in this full QCD simulation is still too heavy to see the decrease. Third, $d_{N}$ does not vanish in the chiral limit due to the lattice artifact that the chiral symmetry is explicitly broken in the Wilson-type quark action. Indeed the topological susceptibility, which is also theoretically expected to vanish in the chiral limit, does not

FIG. 7 (color online). Same as Fig. 5 at $K=0.1374$. 
FULL QCD CALCULATION OF NEUTRON ELECTRIC ...
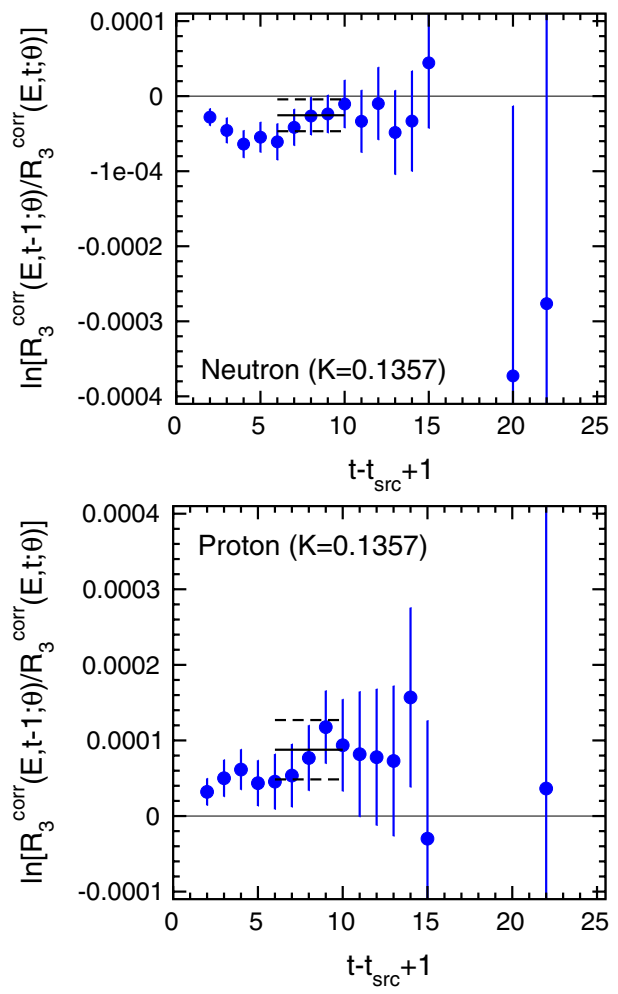

FIG. 9 (color online). The effective mass plot of $R_{3}^{\text {corr }}$ defined in Eq. (14) as a function of time in lattice unit at $K=0.1357$ for neutron (top) and proton (bottom). The solid line denotes the central value of the fitting result and the two dash lines indicate an error band.
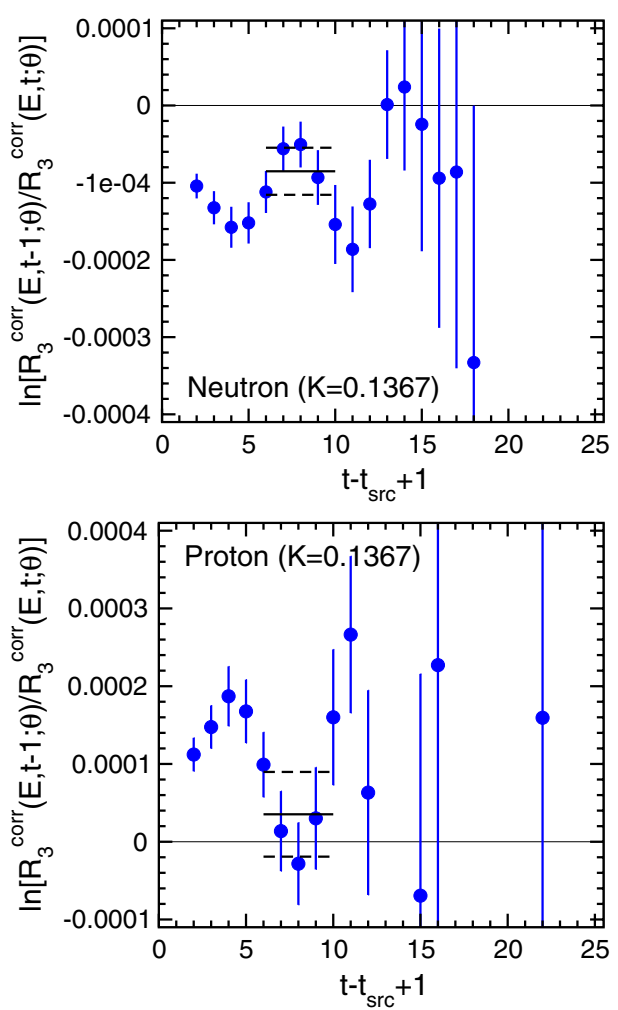

FIG. 10 (color online). Same as Fig. 9 at $K=0.1367$.
PHYSICAL REVIEW D 78, 014503 (2008)
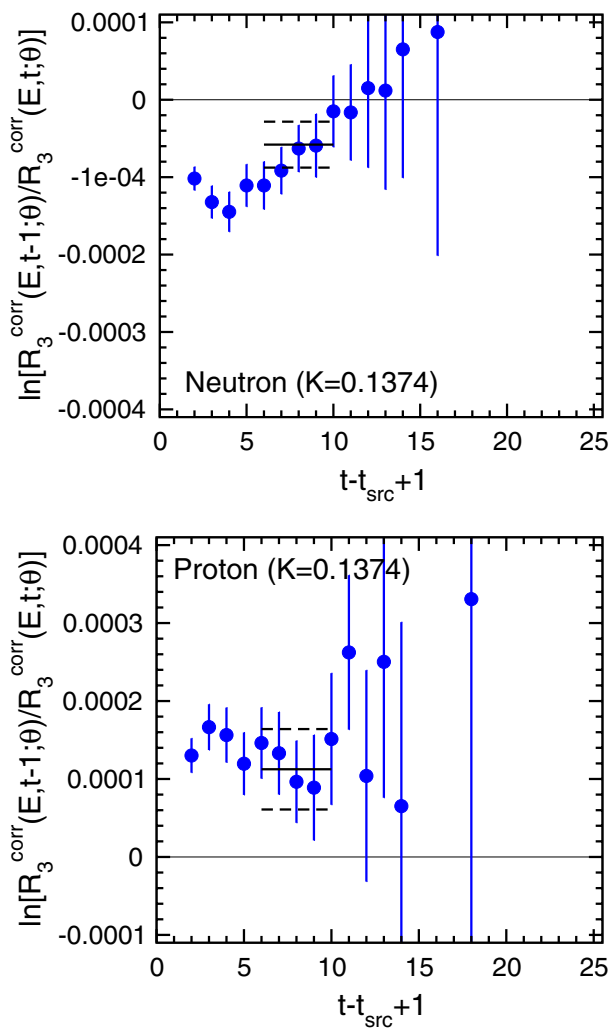

FIG. 11 (color online). Same as Fig. 9 at $K=0.1374$.
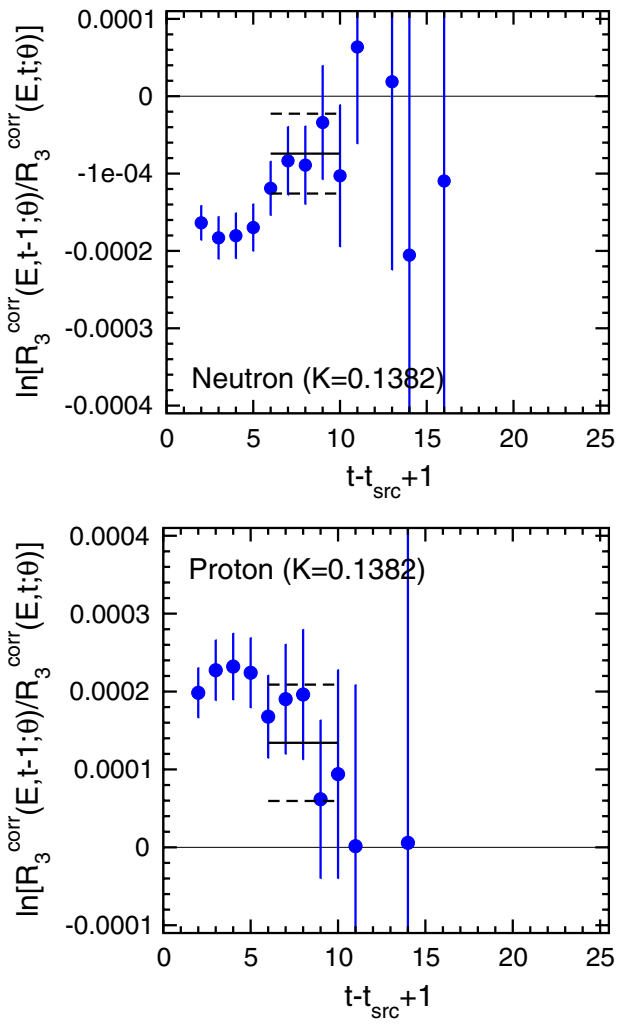

FIG. 12 (color online). Same as Fig. 9 at $K=0.1382$. 
TABLE II. Results for neutron and proton EDM at each quark mass.

\begin{tabular}{lcrr}
\hline \hline$K$ & $m_{\mathrm{PS}}(\mathrm{GeV})$ & Neutron EDM $(\mathrm{e} \cdot \mathrm{fm})$ & Proton EDM $(\mathrm{e} \cdot \mathrm{fm})$ \\
\hline 0.1357 & 1.13 & $-0.014(11)$ & $0.049(21)$ \\
0.1367 & 0.93 & $-0.046(16)$ & $0.019(29)$ \\
0.1374 & 0.76 & $-0.031(16)$ & $0.060(28)$ \\
0.1382 & 0.53 & $-0.040(28)$ & $0.072(49)$ \\
\hline \hline
\end{tabular}

show the decrease in full QCD configurations of this paper [18]. In Fig. 14, $d_{N}$ is plotted as a function of the topological susceptibility instead of the pion mass squared. Contrary to the case of the pion mass squared, the change of the topological susceptibility is too little to observe a possible decrease of $d_{N}$ with large statistical errors. This suggests that the combination of the second and third possibilities is a main reason for the mass dependence of $d_{N}$ in Fig. 13.

We also present the results of the mass dependence for the nucleon $C P$-odd phase factor in full QCD calculation. As discussed in $[12,13]$, the next-leading term in the nucleon spinor structure contains additional phase factor $f_{1}^{N}$, which arises from $m_{N}^{\theta} e^{i f_{N}^{1} \theta \gamma_{5}}=m_{N}\left(1+i f_{N}^{1} \theta \gamma_{5}\right)+$ $\mathcal{O}\left(\theta^{2}\right)$, as
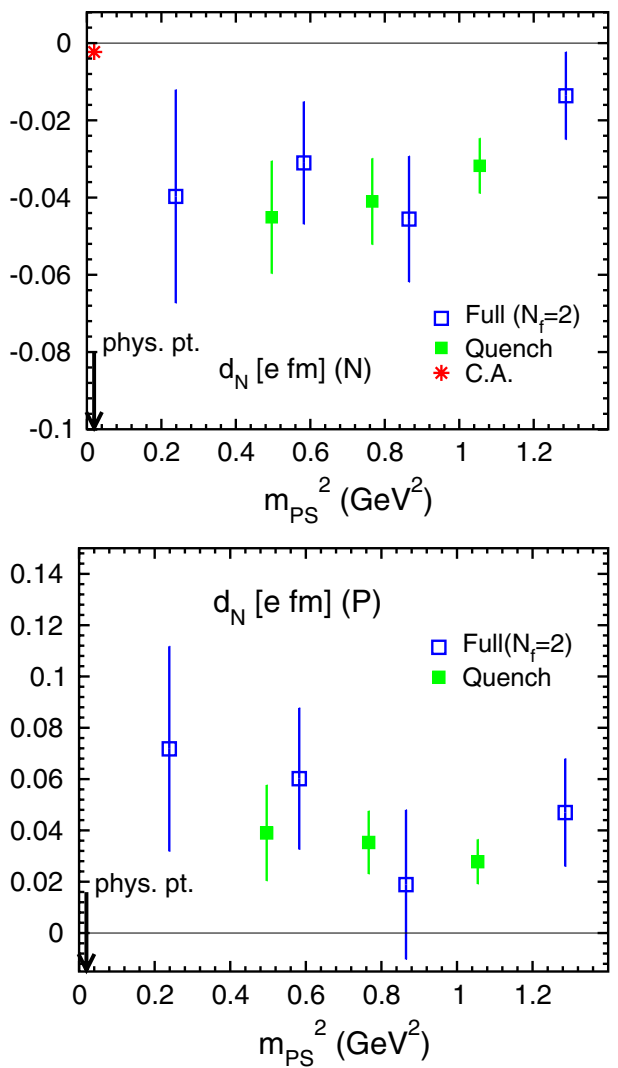

FIG. 13 (color online). EDM as a function of the pseudoscalar meson mass squared $m_{\mathrm{PS}}^{2}$ for neutrons (top) and protons (bottom). The arrow shows the physical point of the pion mass squared, $m_{\pi}^{2}=0.0195 \mathrm{GeV}^{2}$, and the star symbol denotes the result of the current algebra (C.A.) [2].

$$
\langle N(\vec{p}, t) \bar{N}(\vec{p}, 0) Q\rangle=\left|Z_{N}\right|^{2} e^{-E_{N} t} \frac{f_{N}^{1} m_{N}}{2 E_{N}} \gamma_{5},
$$

where $\vec{p}, Z_{N}, E_{N}$ denotes the nucleon momentum, amplitude, and energy, respectively. This factor goes to zero toward the massless limit because of the same reason as NEDM does. In Fig. 15, $f_{N}^{1}$ in full QCD is plotted as a function of $m_{\mathrm{PS}}^{2}$, together with the quenched results. Although the statistical errors are not so large compared to the EDM results, it does not show the expected decrease toward the chiral limit as well as the EDM case. This observation suggests that it is unlikely that the correct chiral behavior of the EDM is hidden in its large statistical errors.

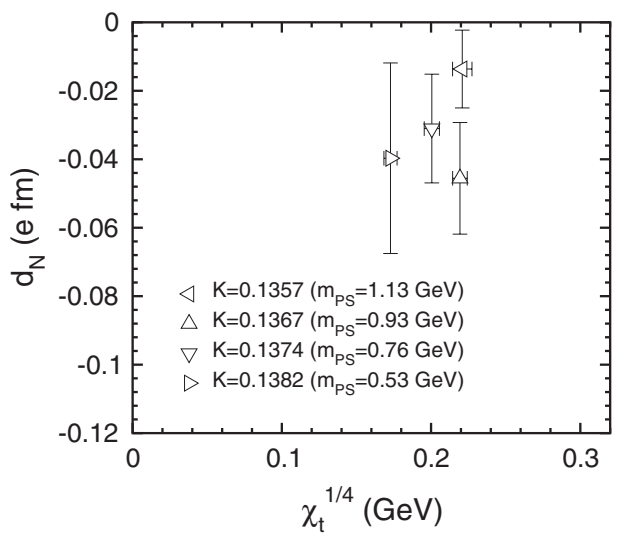

FIG. 14. Neutron EDM as a function of topological susceptibility $\chi_{t}$.

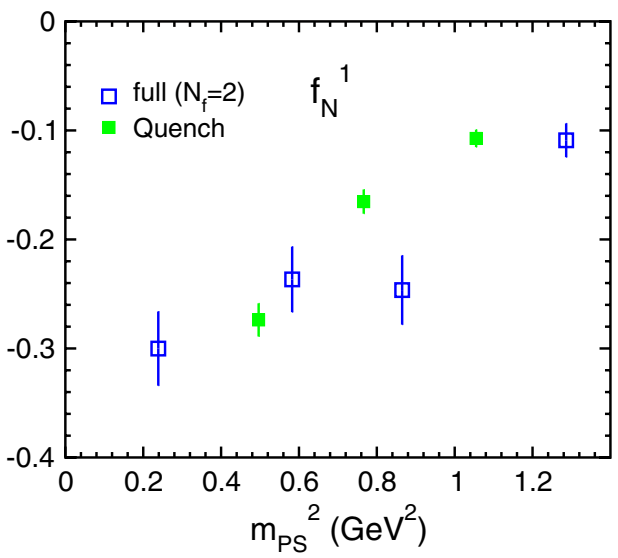

FIG. 15 (color online). $\quad C P$-odd phase factor of the nucleon propagator $f_{N}^{1}$ as a function of the pion mass squared. 


\section{SUMMARY AND DISCUSSION}

In this paper, we present the evaluation of the NEDM in full QCD simulation using the external electric field method. After accumulating a huge number of statistics with multiple sources and an average over forward and backward propagation, we have obtained nonzero value of the nucleon EDM for the first time in full QCD. Statistical errors of the EDM in full QCD are still larger than in the previous quenched case. The mass dependence of the EDM, which is similar to the quenched one, does not show the expected behavior in full QCD that it vanishes towards the chiral limit. Besides the large statistical errors, there may be two main reasons. One is that sea quark masses used in this paper are still too heavy to see the expected decrease, the other is that the explicit chiral symmetry breaking of the Wilson-type quark action spoils the expected chiral behavior. Indeed this chiral behavior of the EDM is very similar to that of the topological susceptibility.

Since it now becomes possible to calculate the EDM in full QCD, we should proceed toward the precise evaluation of the NEDM. First of all, we should further decrease the sea quark mass to clearly observe the chiral behavior of EDM. As reported in the recent advanced work in the PACS-CS collaboration [19], $2+1$ flavors dynamical con- figurations are being generated at the quark mass close to the physical point, and these configurations will be available soon. Second, we should decrease statistical errors of the EDM. For this purpose, it may be better to switch to the form factor method [12], though the zero momentum transfer limit has to be taken in this case. From the previous work [13] and this work, the good chiral behavior of the quark action seems not so relevant to obtain the signal of the EDM, and we are currently calculating the EDM form factor using the clover quark action [20]. Finally, the effect of disconnected loop diagram, ignored in our studies, should also be included in the calculation. A preliminary study shows that it is possible to include this effect with the current available resources [20].

\section{ACKNOWLEDGMENTS}

This work is supported in part by Grant-in-Aid of the Ministry of Education (No. 13135204, No. 15540251, No. 18540250, No. 70447225). Numerical simulations are performed on Hitachi SR11000 at the High Energy Accelerator Research Organization (KEK). At KEK this simulation is under support of the Large Scale Simulation Program (No. 06-04). We use the two-flavor full QCD configurations generated by the CP-PACS Collaboration [14].
[1] P. G. Harris et al., Phys. Rev. Lett. 82, 904 (1999).

[2] R. J. Crewther, P. Di Vecchia, G. Veneziano, and E. Witten, Phys. Lett. B 88, 123 (1979); 91, 487(E) (1980); P. Di Vecchia, Acta Phys. Austriaca Suppl. 22, 341 (1980); K. Kawarabayashi and N. Ohta, Prog. Theor. Phys. 66, 1789 (1981).

[3] S. Aoki and T. Hatsuda, Phys. Rev. D 45, 2427 (1992); H. Y. Cheng, Phys. Rev. D 44, 166 (1991); A. Pich and E. de Rafael, Nucl. Phys. B367, 313 (1991).

[4] M. Pospelov and A. Ritz, Nucl. Phys. B558, 243 (1999); B573, 177 (2000); Phys. Rev. Lett. 83, 2526 (1999); Phys. Rev. D 63, 073015 (2001); Chuan-Tsung Chan, E. M. Henley, and T. Meissner, arXiv:hep-ph/9905317.

[5] J. Gasser and H. Leutwyler, Phys. Rep. 87, 77 (1982); Ann. Phys. (N.Y.) 158, 142 (1984); Nucl. Phys. B250, 465 (1985).

[6] T. Ishikawa et al. (CP-PACS/JLQCD Collaboration), arXiv:0704.1937; C. Aubin et al. (MILK Collaboration), Phys. Rev. D 70, 114501 (2004).

[7] W.-M. Yao et al.(Particle Data Group), J. Phys. G 33, 1 (2006).

[8] R. D. Peccei and H. R. Quinn, Phys. Rev. Lett. 38, 1440 (1977); Phys. Rev. D 16, 1791 (1977); S. Weinberg, Phys. Rev. Lett. 40, 223 (1978); F. Wilczek, Phys. Rev. Lett. 40, 279 (1978); R. D. Peccei, Adv. Ser. Dir. High Energy Phys. 3, 503 (1989).
[9] P. Sikivie, AIP Conf. Proc. 805, 23 (2005) [arXiv:hep-ph/ 0509198], and therein references.

[10] M. Pospelov and A. Ritz, Ann. Phys. (N.Y.) 318, 119 (2005); J. Hisano and Y. Shimizu, Phys. Rev. D 70, 093001 (2004).

[11] S. Aoki and A. Gocksch, Phys. Rev. Lett. 63, 1125 (1989); 65, 1172(E) (1990); 65, 1092 (1990).

[12] E. Shintani et al., Phys. Rev. D 72, 014504 (2005).

[13] E. Shintani et al., Phys. Rev. D 75, 034507 (2007).

[14] A. Ali Khan, et al. (CP-PACS Collaboration), Phys. Rev. D 65, 054505(E) (2002); 67, 059901(E) (2003); http:// www.jldg.org/lqa/.

[15] F. Berruto, T. Blum, K. Orginos, and A. Soni, Phys. Rev. D 73, 054509 (2006).

[16] W. Detmold, B. C. Tiburzi, and A. W.-Loud, Phys. Rev. D 73, 114505 (2006).

[17] P. Weisz, Nucl. Phys. B212, 1 (1983); P. Weisz and R. Wohlert, Nucl. Phys. B236, 397 (1984); B247, 544(E) (1984); M. Lüscher and P. Weisz, Commun. Math. Phys. 97, 59 (1985); 98, 433(E) (1985).

[18] A. Ali Khan et al. (CP-PACS Collaboration), Phys. Rev. D 64, 114501 (2001).

[19] Y. Kuramashi et al. (PACS-CS Collaborations), Proc. Sci., LAT2006 (2006) 029; Y. Kuramashi, Proc. Sci., LATTICE2007 (2007) 017.

[20] E. Shintani, S. Aoki, and Y. Kuramashi (unpublished). 